\title{
Review article: pulmonary embolism in pregnancy: suspicion, diagnosis and therapy
}

\begin{abstract}
During pregnancy the haemostatic system is progressively activated to prepare the haemostatic needs of delivery and postpartum. Blood hypercoagulability, endothelial injury and stasis of blood flow predispose to thrombus formation.

Pulmonary embolism is a complication of venous thrombosis and is a leading cause of pregnancy related deaths at any trimester. It is extremely common disorder without specific clinical features.

The physician is called to suspect the disorder and give a prompt diagnosis and therapy to avoid the lethal condition. Delays in pulmonary embolism diagnosis and therapy are common and represent an important issue.

Keywords: pulmonary embolism, venous thromboembolism, subsegmental emboli, pregnancy, mortality, epidemiology, risk factors, diagnosis, arterial blood-gases, electrocardiogram, ventilation perfusion scan, computed tomography, pulmonary angiogram, magnetic resonance, compression ultrasonography, echocardiogram, D-dimers, troponin, brain natriuretic peptide, fetal radiation, breast radiation, radiation exposure, thrombolysis, heparin, low molecular weightheparin, anticoagulant, prevention, thromboprophylaxis, vena caval filters
\end{abstract}

Volume 10 Issue I - 2019

Irene M Orfanoudaki

Obstetrics Gynecology, University of Crete, Greece

Correspondence: Irene M Orfanoudaki, Obstetrics Gynecology, University of Crete, 22, Arhiepiskopou Makariou STR, Greece, Tel +306945268822, +302810343376, Email orfand_e@otenet.gr, eirni.m.orfanoudakis@gmail.com

Received: December 09, 2018 | Published: January 03, 2019
Abbreviations: PE, pulmonary embolism; IVC, inferior vena cava; LMWH, low-molecular-weight heparin; UFH, unfractionated heparin

\section{Introduction}

Pulmonary embolism in pregnancy is a cause which leads to death throughout the duration of pregnancy. Pulmonary embolism is a complication of disturbance of coagulation which leads to thromboembolic disease. The symptoms and signs of pulmonary embolism are no specific in pregnant woman and the diagnosis ${ }^{1}$ may be delayed or missed.

An embolic episode follows the missed clinical condition and one third of patients die. A sudden death may be occurred after a massive pulmonary embolism. It is crucial to understand the oncoming disturbance of coagulation, to prevent thromboembolic disease and efficacious prophylactic treatment to be used. ${ }^{1}$

\section{Etiology}

Pulmonary thromboembolism is a disturbance of formation of thrombus during pregnancy. In this period, the haemostatic system changes progressively to carry out the delivery. The gravid uterus and enlarging iliac arteries compress the venous system and a dilation of lower extremities is occurred. At any time of pregnancy and delivery, endothelial injury may transpire ${ }^{2}$ and the endogenous thrombolytic system forms and lyses microthrombi in the venous system.

Normal pregnancy and the puerperium, are associated with a hypercoagulable state, because fibrinogen and factors II, VII, VIII, X and $\mathrm{XII}^{3-5}$ increase and lead to enhanced thrombin production (Figure $1){ }^{6}$

As gestation increases levels of the (anticoagulant) protein $\mathrm{S}$, decline $^{5}$ and resistance is increased for activation of protein $\mathrm{C}$ in the last trimesters of pregnancy.

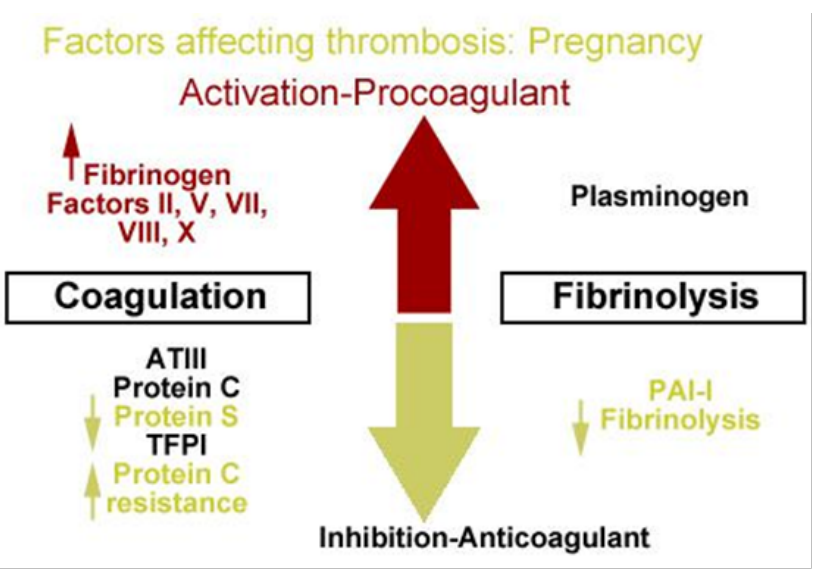

Figure I Thrombus factors which change during pregnancy. ${ }^{10}$

The acute phase of response has as a result to activate platelets, to increase fibrin generation and decrease fibrinolytic activity mostly in the third trimester. In a decreased fibrinolytic state ${ }^{6}$ contribute serum plasninogen activator inhibitor-1 (PAI-1) and placental PAI-2.This fibrinolytic state increase during pregnancy.

Heterozygous carriers of Factor $\mathrm{V}^{7}$ or prothrombin mutation ${ }^{8}$ as well as women with antisphospolipid syndrom present high risk of pregnancy -related thrombosis. ${ }^{9}$

Uteroplacental thrombosis and recurrent pregnancy loss are associated with an increase of phosphatidylserine which is expressed in tissue, and produces phosphatidylserine microparticles and endothelial microparticles. If these particles remain in increased levels at least 3 months after pregnancy loss, then a chronic endothelial damage is presented and a next pregnancy may stop.

The physical and hormonal changes associated in pregnancy contribute in hyper coagulation in pregnant women. Hormonal factors 
produce stasis of blood which leads to venodilation of pregnancy. An increased stasis ${ }^{3,4}$ is produced by progesterone levels which are elevated at the starting of pregnancy, and produce venous distensibility and capacity.

As pregnancy goes on, the uterus which enlarges compress the common iliac vein. ${ }^{4}$ The significant venous stasis is observed more common in the left deep system because the right iliac artery compresses the left common iliac vein. ${ }^{3}$

During this venous stasis, thrombi originate in venous valve pockets. Most common these thrombi (emboli) can arise from deep venous system, rarely from right heart chambers or other veins. But, they can originate from any part in the body.

These emboli, travel with blood and reach to the cavities of the heart, right atrium, right ventricle, left atrium, left ventricle and finally to the lungs. In the lungs, if emboli are massive, they produce hemodymamic compromise because the lodge at the bifurcation of the main pulmonary artery of the lobar branches.

When thrombi are smaller, they are able to travel far away and occlude the smaller vessels in the periphery of the lungs. A pleuritic chest pain is present due to an inflammatory response of parietal pleura. The emboli mostly are multiple and are often to the upper lobes of the lungs (Figure 2). ${ }^{10}$

\section{Incidence}

The lung embolism is observed five times more frequently in pregnant women than in non pregnant women and its frequency is 1 in 200 deliveries to 1 in 1400 deliveries, while deaths occur 1-2 cases in 100.000 pregnant women. Its relative risk is 4.29 and incidence is 199.7 per 100.00 cases.

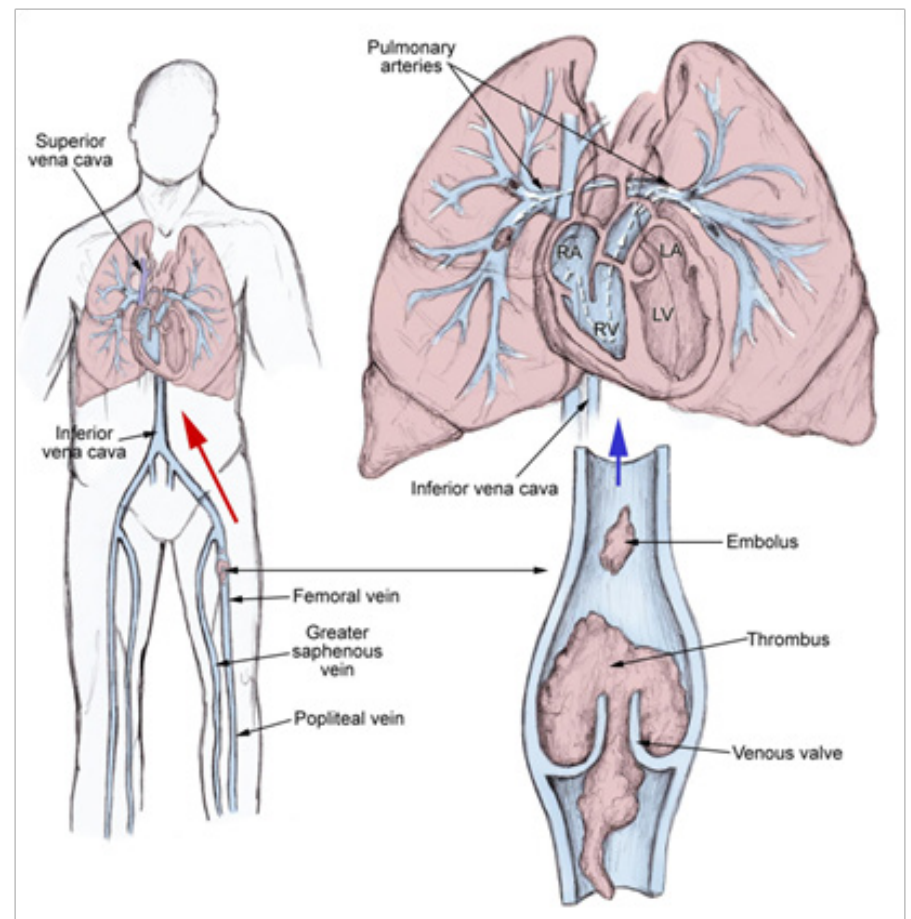

Figure 2 The etiology of pulmonary embolism. ${ }^{10}$

During pregnancy there is low rate of pulmonary embolism (10.6 incidents per 100.000 women), in postpartum period the rate is higher (159.7 incidents per 100.000 women). ${ }^{11}$ In many countries the incidence may differ depending mainly in accuracy of diagnosis than the actual incidence (Figure 3).

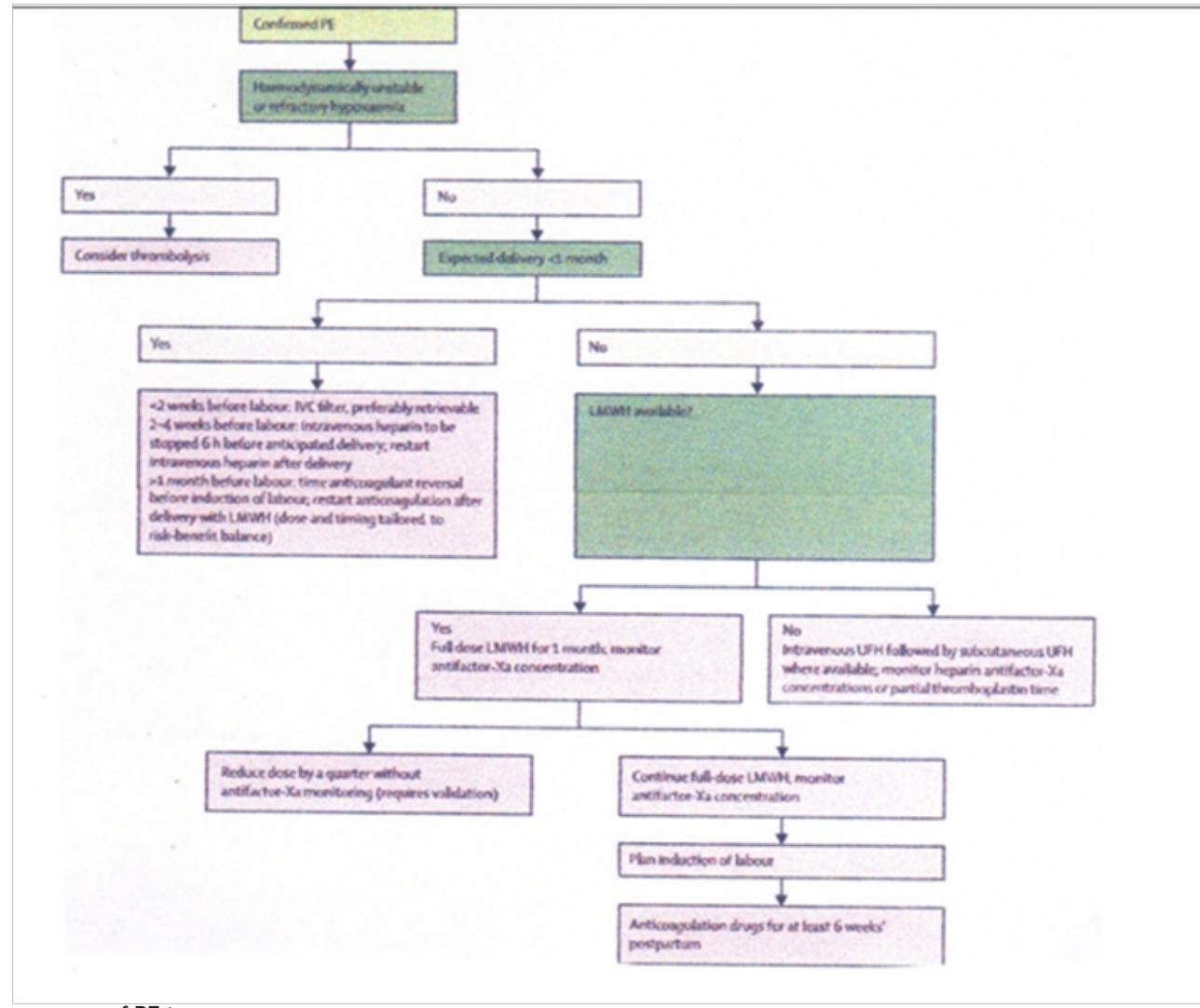

Figure 3 Suggested treatment of PE in pregnancy. 
Black women appear to have significantly higher incidence of pulmonary embolism than white ones. ${ }^{12}$

\section{Diagnosis}

When a pulmonary embolism is apparent, is discerned as acute or chronic

If the thrombus remains in the vascular lumen centrally, occludes the diameter of vessel and distends the involved vessel, then pulmonary embolism is characterized as an acute.

If the thrombus remains eccentric and contiguous to the wall of the vessel, the diameter of the vessel is reduced more than $50 \%$, is observed in the arterial web and is obvious the recanalization within the embolus, then the pulmonary embolism is characterized as chronic.

The site of thrombus inside the arterial branch sets the diagnosis as central or peripheral.

The central pulmonary embolism includes major vessels as the main pulmonary, the left and right pulmonary arteries, the anterior trunk, the right and left interlobar arteries, the left upper lobe trunk, the right middle lobe artery and the right and left lower lobe arteries.

The peripheral pulmonary embolism include the segmental and subsegmental arteries of the right upper, middle and lower lobe, the lingula, the left upper and lower lobe.

If a pregnant woman is admitted in hospital with a hemodynamic compromise and a pulmonary embolus is present in both pulmonary arteries then the pulmonary embolism is characterized as massive. After a sudden death, autopsy shows massive pulmonary embolism in $80 \%$ of pregnant patients, and after the sudden cardiac death, it is the second cause of death in pregnancy.

These cases present mortality between $30 \%$ and $60 \%{ }^{13-15}$ and 95.5 $96 \%$ of patients present low systolic arterial pressure (lower or equal to $90 \mathrm{~mm} \mathrm{Hg}) .{ }^{16,17}$

Massive pulmonary embolism is associated with hemodynamic parameters and clinical picture of myocardial injury rather than to anatomic findings because of it is characterized from adverse outcomes..$^{18}$

The clinical picture of pulmonary embolism presents a woman in shock. The main signs are hypotension, tachypnea, tachycardia and poor perfusion of extremities. The physician may also discern a woman with impaired mentation, weak, pale, sweaty and oliguric.

These patients may present all signs of pulmonary hypertension and a high index of suspicion is required because these signs are not specific for pulmonary embolism. These signs include palpable impulse over the second left intercostal space, loud P2, right ventricular S3 gallop, a systolic murmur louder on inspiration at left sternal border.

When a pregnant woman is admitted with acute onset of pleuritic chest pain, breathlessness, without or with hemoptysis an embolus has produced acute occlusion of a pulmonary artery leading to the parenchymal infraction. The clinical picture is characteristic of an acute pulmonary infraction. The ECG findings are normal and myocardial pain has no response to nitroglycerin. The breath sounds are diminished and dullness to percussion may be detected. ${ }^{10}$ Pleural friction rub may be audible or palpable and an involved hemithorax may be not detected because of the localized tenderness.

When an acute pulmonary embolism is appeared without peripheral occlusion of the vessel there are not specific physical signs. Pleuritic pain sometimes may be present, tachypnea and tachycardia frequently are detected, at the point of thrombosis crackles may be heard and rarely local wheeze. ${ }^{10}$

In diagnosis and care of pulmonary embolism it is very important for the initial physician to suspect and have a systematized and aggressive evaluation as well a plan of treatment because the majority of deaths occur in the first 1-2 hours of care.

The anticoagulant therapy has diminished the mortality rate of lung thrombosis. In non massive lungs thrombosis, the death is lower than $5 \%$ in the first 6 months of therapy. Recurrence may observe in less than $5 \%$ and reaches $30 \%$ after 10 years. ${ }^{19}$

\section{Clinical signs and symptoms for pulmonary embolism are nonspecific}

An abrupt onset of pleuritic chest pain may be the unique first symptom. A hypoxia and shortness of breath may coexist. The physician may detect gradually dyspnea to sudden hemodynamic collapse. There no obvious symptoms at examination.

The symptoms may be atypical and include: pleuritic chest pain, dyspnea, hemoptysis, frank pain, productive cough, seizures, syncope, abdominal pain, fever, wheezing, decreasing level of consciousness, new onset of atrial fibrillation.

The most common symptoms of pulmonary embolism include: ${ }^{20}$ Dyspnea (73\%), Pleuritic chest pain (66\%), cough (37\%), Hemoptysis $(13 \%)$. The pulmonary embolism may present with pleuritic chest pain, the sole physical finding, without other symptoms or risk factors and is a particularly worrisome symptom. The rate of this symptom is $84 \%$ of patients. The pleuritic chest pain indicates that thrombus is smaller and the obstruction is peripherally.

When a pulmonary thrombosis is established the signs include: tachypnea $96 \%$, rates $58 \%$, accentuated second heart sound $53 \%$, tachycardia $44 \%$, fever $43 \%$, diaphoresis $36 \%$, S3 or S4 $34 \%$, clinical signs and symptoms suggesting thrombophlebitis $32 \%$, lower extremity edema $24 \%$, cardiac murmur $23 \%$, cyanosis $19 \%$.

For establishing or excluding the diagnosis of pulmonary embolism the pregnant patient must undergo on specific diagnostic tests because the routine laboratory findings are not specific for confirmation the diagnosis.

\section{Physical examination}

The physical findings are quite variable depending on the category of pulmonary embolism.

Four categories of pulmonary embolism are discerned: Massive lung thrombosis, acute lung infarction, acute lung thrombosis without infarction, multiple lung thrombi.

In massive lung thrombosis, patients are admitted to hospital in shock with systematic hypotension, poor perfusion of the extremities, tachycardia, and tachypnea. Also they are appeared weak, pale, sweaty and oliguric with impaired mentation. Pulmonary hypertension may be present. Hemodynamic parameters of myocardial injury rather than anatomic findings may define massive pulmonary embolism. ${ }^{18}$

In acute lung infarction, patients present acute onset of pleuritic chest pain, breathlessness and hemoptysis. May be there is difficulty in the distinction of the chest pain from ischemic myocardial pain. Signs of pleural effusion may be present. ${ }^{10}$ 
In acute lung thrombosis without infarction patients present non specific physical signs. Tachypnea, tachycardia may be detected, pleuritic pain sometimes may be present, crackles may be heard at the points of thrombosis and local wheeze may be heard rarely. ${ }^{10}$

In multiple lung thrombi patients present physical signs of pulmonary hypertension and cor pulmonale. ${ }^{10}$ These findings include: elevate venous pressure, present palpable impulse over the second left intercostal space, loud P2, right ventricular S3 gallop, asystolic murmur louder on inspiration at left sternal border, hepatomegaly, ascites and edema.

\section{Pathophysiology}

A thrombus or an embolus is formed when there is hypercoagulability of blood, stasis or turbulence of blood flow and endothelial injury. All these factors are well known as: The Virchow triad. ${ }^{21-23}$

Table I The Causes for pulmonary embolism are multifactorial ${ }^{10}$

\section{Pulmonary embolism produces respiratory and hemodynamic consequences}

In acute respiratory consequences are included hyperventilation, hypoxemia, and alveolar dead space, regional loss of surfactant and lung infarction. Ventilation-perfusion mismatch, intrapulmonary shunts, reduced cardiac output, and intracardiac shunt are the mechanisms of hypoxemia. The cross-sectional area of the pulmonary vascular bed is reduced resulting in an increment in pulmonary vascular resistance and the right ventricular afterload is increased. If the afterload is increased severely, right ventricular failure may ensue. The humoral and reflex mechanisms contribute to the pulmonary arterial constriction.

As the therapy is initiated the resolution of thrombi occurs during the first 2 weeks. When the initial thrombus failures to undergo lyse or a recurrent thrombus appears chronic pulmonary hypertension may establish (Tables $1-3) .{ }^{10}$
Venous stasis
Hypercoagulable
Obesity, trauma, surgery
Immobilization
Surgery and trauma
ocal venous stasis, accumulation of clotting factors and fibrin
Activating clotting factors
Pregnancy
Oral contraceptives and estrogen replacement
Malignancy
Hereditary factors
Acute medical illness
Additional risk factors
Estrogen-containing birth control pills
Solid tumors, leukemias, lymphomas
pancreatic carcinoma, brochogenic carcinoma, carcinomas of the genitourinary tract, colon, stomach and breast
Antithrombin III deficiency, Protein C deficiency, Protein S deficiency, Factor V Leiden, Plasminogen abnormality, Plasminogen activator abnormality, Fibrinogen abnormality, Resistance to activated protein $\mathrm{C}$
Aids (lupus anticoagulant), Behcet disease, Congestive heart failure, Myocardial infarction, Polycythemia, Systemic lupus erythematosus, Ulcerative colitis
Drug abuse, Drug-induced lupus anticoagulant, Hemolytic anemias, Heparin-associated thrombocytopenia, Homocystinemia, Homocystinuria, Hyperlipidemias, Phenothiazines, Thrombocytosis, Varicose veins, Venography, Venous pacemakers, Venous stasis, Warfarin (first few days of therapy), inflammatory bowel disease

Risk factors for pulmonary embolism. ${ }^{24}$

Table 2 One or more risk factors for pulmonary embolism ${ }^{24}$

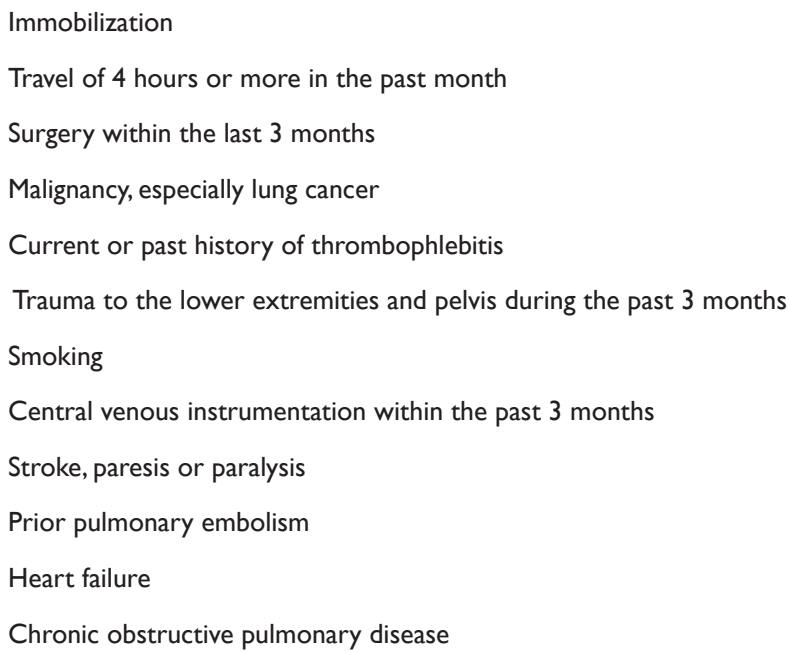


Table 3 Risk factors for VTE in pregnancy ${ }^{25}$

\begin{tabular}{|c|c|c|}
\hline Pre-existing & New onset/transient & Obstetric \\
\hline Previous VTE & Early pregnancy & Antenatal \\
\hline Heritable thrombophilia & a. Hyperemesis gravidarum & a. Multiple pregnancy \\
\hline \multirow{2}{*}{ Acquired thrombophilia } & \multirow{2}{*}{ b. Ovarian hyperstimulation syndrome } & b. Assisted reproduction \\
\hline & & Therapy \\
\hline Family history of VTE & Throughout pregnancy & a. Pre-eclampsia \\
\hline $\begin{array}{l}\text { Medical co-morbidities (including SLE, } \\
\text { nephrotic syndrome, sickle cell disease, } \\
\text { cancer, inflammatory conditions) }\end{array}$ & $\begin{array}{l}\text { a. Surgical Procedures (inc. ERPC, postpartum steri- } \\
\text { lisation) }\end{array}$ & Delivery \\
\hline Age $>35$ years & b. Admission & a. Caesarean section \\
\hline $\mathrm{BMI}>30 \mathrm{~kg} \cdot \mathrm{m}^{-2}$ & c. Immobility (e.g. symphysis pubis dysfunction) & b. Prolonged labour \\
\hline Parity $\geq 3$ & c. Dehydration & c. Midcavity rotational forceps delivery \\
\hline Smoking & Systemic Infection & Postnatal \\
\hline Varicose veins & a. Travel of duration $>4 \mathrm{hrs}$ & a. Postpartum haemorrhage (>I litre) \\
\hline Paraplegia & & b. Blood transfusion \\
\hline
\end{tabular}

\section{Diagnostic tests}

Non-imaging methods: Detection of pulmonary embolism with routine laboratory findings is difficult because of them are nonspecific and are not helpful.

After a detailed history of the patient, if no obvious cause for embolic disease is apparent must be checked the deficiency of antithrombin III, protein $\mathrm{C}$ or protein $\mathrm{S}$, lupus anticoagulant, homocysteinuria, occult neoplasm and connective tissue disorders.

\section{Laboratory tests in patients with suspected pulmonary embolism include}

D-dimer testing: may be the next step ${ }^{26}$ when a diagnostic dilemma is occurred. Negative results exclude pulmonary embolism. Its sensitivity is 0.95 and negative ratio is $0.13 .{ }^{27}$ During pregnancy and the postpartum period the specificity of D-dimer testing might be poor. For a suspected DVT in pregnancy the negative predictive value of D-dimer testing is high. ${ }^{28}$ During pregnancy, D-dimer concentration rises gradually and drops rapidly in the immediate postpartum period. Normal levels returns after 4-6 weeks of postpartum. ${ }^{29}$

Ischemia-modified albumin level (IMA): 93\% sensitive and 75\% specific for pulmonary embolism. ${ }^{30}$

White blood cell count: It is noticed to be normal or elevated.

Arterial blood gases: A low PO2 (hypoxemia) is a strong predictive index for pulmonary embolism.

Serum troponin levels: More of $50 \%$ of patients with a moderate pulmonary embolism may elevate the serum troponin levels because of the acute right ventricular myocardial stretch. ${ }^{31}$ Increased mortality in pulmonary embolism is correlated with elevated troponin levels. ${ }^{32,33}$

Brain natriuretic peptide (BNP): High levels of BNP or its precursor, N-terminal pro-brain natriuretic peptide (NT-proBNP), in acute lung thrombosis, are correlated with increased risk of subsequent complications and mortality. ${ }^{34,35}$ BNP testing despite is not currently recommended may provide prognostic information in acute pulmonary embolism. ${ }^{36}$

\section{Imaging studies}

It is well known that radiation is associated with teratogenic and oncogenic effects to the fetus-human being and the imaging studies for diagnosis during pregnancy need careful evaluation. It has yet not established the minimum dose of radiation which produces an obvious increased risk of teratogenicity to the human fetus.

Any exposure to radiation above the natural background radiation produces an increased risk of chlidhood malignancy ${ }^{37}$ due to a different threshold effect in oncogenicity from in-utero radiation exposure.

An exposure of the conceptus to 0.01 Gy above natural background radiation has as a result the increase of probability of cancer from $0.03 \%$ to $0.04 \%$ before the age of 20 years. ${ }^{26}$

The total radiation in which a fetus is exposed after a chest radiograph, ventilation perfusion scan, and conventional pulmonary angiogram combined with CT pulmonary is $0.004 \mathrm{~Gy}{ }^{38-40}$

Fetal malformations have a threshold of $100-200 \mathrm{mGy}^{41}$ $1000 \mathrm{mGy}=100 \mathrm{rad}$. Radiation of the fetus at any time during gestation age with $0.1 \mathrm{~Gy}$ is considered a threshold beyond congenital abnormalities is possible. ${ }^{38-40}$

Despite of these, pulmonary embolism in pregnancy which remains untreated presents mortality which outweight the oncogenic and teratogenic risk of fetus radiation.

Computed tomography angiography (CTA), Spiral (helical) CT scanning is put in practice in up to $57 \%$ of patients. ${ }^{42}$ The criterion standard for diagnosing pulmonary embolism in pregnant women is Multidetector-row CTA (MDCTA) ${ }^{43}$ This test is able to be performed with safety in all trimesters of pregnancy. ${ }^{44}$ The fetus radiation is related on gestation age and the distance of the uterus from the thorax, varies from 0.00033 to $0.01308 \mathrm{rad}^{44}$ and it has sensitivity $57-100 \%$ and specificity $64-100 \%{ }^{45,46}$

Pulmonary angiography: When MDCTA is not available, pulmonary angiography consists the standard criterion for the diagnosis of pulmonary embolism. 
Chest radiography: It is a nonspecific test. In most cases of pulmonary embolism, chest radiography is abnormal. ${ }^{47}$

ECG: $20 \%$ of patients with proven pulmonary embolism present disturbances in ECG, such as tachycardia and nonspecific ST-T wave abnormalities.

V/Q scanning: It is recommended when CT scanning is not available. ${ }^{48}$

MRI: Presents a sensitivity $85 \%$ and specificity $96 \%$. MRI gives increased signal intensity within the pulmonary artery of pulmonary emboli. ${ }^{49}$

Echocardiography: This method has 59\% and 77\% sensitivity and specificity respectively and central pulmonary embolism can be detected with transesophageal echocardiography. ${ }^{50}$

Venopraphy: The criterion standard test in diagnosis of deep venous thromboembolism (DVT)
Duplex ultrasonography: Demonstrates a DVT at any site. It gives a sensitivity of $50 \%$, specificity of $98 \%$ of right ventricular dilatation and $88 \%$ positive and negative predictive values in diagnosis of pulmonary embolism (Table 4) (Table 5). ${ }^{37}$

Table $4 \mathrm{~A}$ summary of the estimated fetal exposures for the different types of radiological investigations used to diagnose VTE in pregnancy ${ }^{41}$

\begin{tabular}{ll}
\hline Unilateral venography (no abdominal shield) & $3 \mathrm{mGy}$ \\
Limited venography & $<0.5 \mathrm{mGy}$ \\
Perfusion scan (technetium-99m/ I-2mCi) & $<0.12 \mathrm{mGy}$ \\
Ventilation scan (varies with isotope) & $<0.35 \mathrm{mGy}$ \\
CTPA & $0.5 \mathrm{mGy}$ \\
Chest radiography & $<0.1 \mathrm{mGy}$
\end{tabular}

Table 5 Advantages and disadvantages of imaging techniques in pregnancy ${ }^{51}$

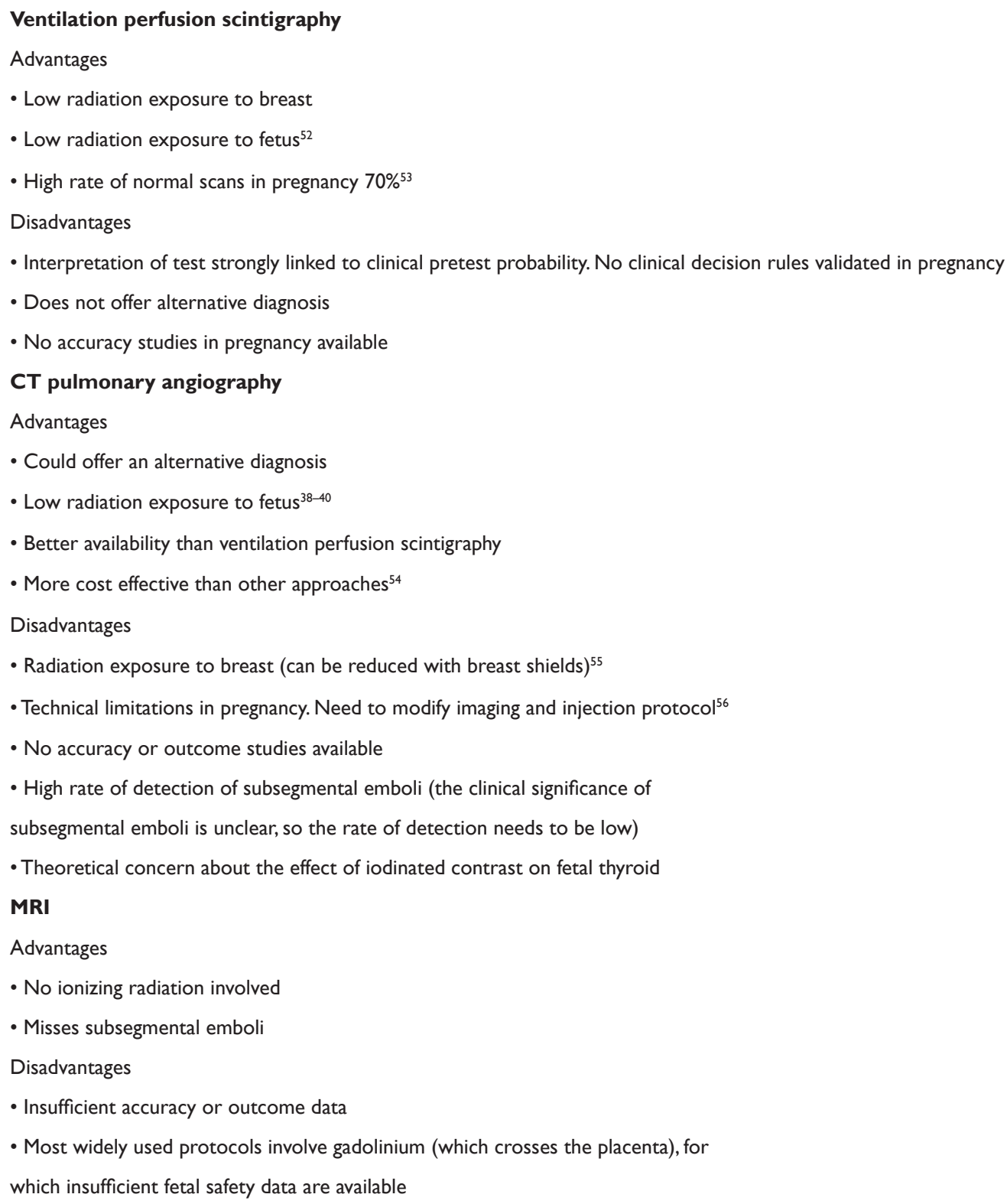


Table Continued....

\author{
Compression ultrasonography \\ Advantages \\ - No exposure to radiation \\ - Non-invasive \\ Disadvantages \\ - Possible low sensitivity in patients without signs and symptoms of deep vein thrombosis
}

\section{Differential diagnosis}

The physician must be very suspicious when a pregnant woman is admitted with severe clinical picture because of the differential diagnosis is extensive (Table 6) (Table 7). ${ }^{10}$

\section{Medical Management of PE Embolism}

If A pregnant woman is admitted with symptoms and signs which arise the suspicion for acute lung thrombosis empiric anticoagulation must be given before the final diagnosis unless there are other contraindications. When the suspicion for PE or DVT is low or intermediate therapy may wait until the final conclusions of diagnostic tests. If the diagnosis of PE or DVT is established therapeutic anticoagulation must be started (Table 8) (Table 9)..$^{57}$

The preferred drugs for therapeutic anticoagualation in pregnancy are heparins. Heparins don't produce teratogenesis of the fetus or fetal hemorrhage, because they do not cross the placenta. ${ }^{58}$

Table 6 Differential diagnosis of pulmonary embolism ${ }^{10}$

\title{
Differential diagnosis of pulmonary embolism
}

\begin{tabular}{|c|c|c|}
\hline - Musculoskeletal pain & - Silicone pulmonary embolism & - Idiopathic pulmonary arterial hypertension \\
\hline - Pleuritis & - Lung trauma & - Pulmonary arteriovenous fistulae \\
\hline - Pericarditis & - Mediastinitis & - Restrictive cardiomyopathy \\
\hline - Salicylate intoxication & -Acute sickle cell disease & - Nonidiopathic pulmonary hypertension \\
\hline - Hyperventilation & - Angina Pectoris & - Sudden Cardiac death \\
\hline - Acute coronary syndrome & - Anxiety Disorders & - Syncope \\
\hline - Acute pericarditis & - Aortic Stenosis & \\
\hline - Acute Respiratory Distress Syndrome & -Atrial Fibrillation & \\
\hline - Cardiogenic Shock & - Dilated Cardiomyopathy & \\
\hline - Cor Pulmonale & - Hypersensitivity Pneumonitis & \\
\hline - Emphysema & - Mitral Stenosis & \\
\hline - Fat embolism & - Myocardial infraction & \\
\hline - Superior vena cava syndrome in emergency medicine & - Pneumothorax imaging & \\
\hline
\end{tabular}

Table 7 Complications of Pulmonary Embolism ${ }^{10}$

\section{Complications of pulmonary embolism}

$\begin{array}{ll}\text { - Sudden cardiac death } & \text { - Severe hypoxemia } \\ \text { - Obstructive shock } & \begin{array}{l}\text { - Right-to-left } \\ \text { intracardiac shunt }\end{array} \\ \text { - Pulseless electrical activity } & \text { - Lung infraction } \\ \text { - Atrial or ventricular arrythmias } & \text { • Pleural effusion } \\ \text { - Secondary pulmonary arterial hypertension } & \text { - Paradoxical embolism } \\ \text { - Thrombophlebitis } & \text { - Heparin-induced } \\ \text { thrombocytopenia }\end{array}$

Table 8 Indications for Antithrombotic Use ${ }^{10}$

- Acute and chronic VTE (including pulmonary embolism)

- Atrial fibrillation

- Valvural and structural heart disease

- Ischemic stroke

- Acute coronary syndromes

- Peripheral artery occlusive disease

Table 9 Indications for Antithrombotic Use in Pregnancy ${ }^{5}$

\section{Treatment of acute VTE}

- Valvular heart disease

- antithrombin deficiency

- antiphospholipid antibody (APLA) syndromes

- other thrombophilias, who have had a prior VTE 
Coumarine derivatives cross the placenta and are classified as category $\mathrm{X}$ by FDA. Warfarin can cause fetal malformations. Unfractionated heparin (UFH) is classified as category $\mathrm{C}$ and low molecular weight heparine (LMWH) as category B drug in pregnancy. Heparins activate antithrombin III preventing the conversion of fibrinogen to fibrin. The thrombus cannot be dissolved by heparins. The fibrinolysis with heparins is safe in pregnancy, but the major risks of fibrinolysis are maternal. The risks are: uteroplacental bleeding ${ }^{59}$ hemorrhage, thrombocytopenia, and osteopenia. Pregnant women have the same risk of bleeding produced by heparin as non pregnant women. ${ }^{58,59}$

With the first suspicion of PE or DVT fibrinolysis should be administered with full-dose LMWH or full-dose unfractionated IV heparin. The therapy must be given throughout the pregnancy, continuing for 4-6 weeks postpartum and totally for at least 6 months.

Despite the anticoagulation of PE and DVT may recur or extend, the mortality rates are reduced $30 \%$ to less than $10 \%$, using heparins.

Comparing with unfractionated heparin, several LMWH products have been found safer and more effective for prophylaxis and for treatment of PE and DVT.

\section{Complications of Medical Management}

When anticoagulation therapy is initiated, patients should be monitored for venous thromboembolism, bleeding (unfractionated heparin $)^{60}$ and allergies associated with heparins, including heparin -induced thrombocytopenia (HIT

\section{Heparin is able to produce thrombocytopenia in two types}

Heparin-associated thrombocytopenia: It is independent from heparin antibodies and it is not immune mediated. It occurs four days after heparin administration and affects $30 \%$ of patients. In this type, heparin produce a direct effect to platelet aggregation and the abnormality of platelet is usually mild, reversible, and self-limited even with the continuation of heparin..$^{59}$

Heparin-Induced Thrombocytopenia (HIT): This type is produced by $\operatorname{IgG}$ antibodies to heparin presence, ${ }^{61,62}$ is immune mediated and may present asymptomatically, or may cause a consumptive thrombocytopenia. This is a very serious complication, in the presence of thrombosis (HIT-thrombosis or HITT). The mortality of HITT without treatment is $20-30 \%$, and may cause significant morbidity from arterial and venous thrombosis. ${ }^{60}$

Heparin and LMWH have no statistically significant difference in appearance of HIT. ${ }^{63,64}$ In the incidence of HIT, the long duration of therapy may be an important factor. ${ }^{65}$ HIT monitoring depends on the clinical circumstances. On therapeutic anti-coagulation with UFH, platelets must be checked every other days 4 to 14 after initiation of therapy. When heparin therapy is stopped, platelet monitoring may stop. The LMWH don't require platelet monitoring. ${ }^{66}$

Heparin produce inhibition of formation of 1,25 dihyd roxyvitamin $\mathrm{D}$, the active metabolite of vitamin $\mathrm{D}^{67}$ and is associated with osteopenia in pregnant women. In large meta-analyses LMWH decreases the risk of mortality, recurrent VTE, and hemorrhage compared with heparin (UFH). ${ }^{60,68}$

Low molecular weight heparins (LMWHs): LMWHs don't cross the placenta during pregnancy, the administration is safe as LMWHs have no risk of fetal teratogenesis or hemorrage, however the efficacy and dose in pregnancy depends on the case and the stage of pregnancy.

During pregnancy the plasma volume increases, the glomerular filtration increases as well renal clearance, the circulating levels of heparin-binding proteins are increased and the degradation by the placenta is increased leading to increased requirements of Heparin. ${ }^{69,70}$ The increased clearance and degradation during progress of pregnancy may diminish the effectiveness of LMWHs. In pregnancy may be required LMWHs in higher doses to achieve target anti-Xa activity. ${ }^{71}$

Estimation of the LMWH administration is performed by monitoring anti-Xa levels depending on pharmacokinetics of the increased plasma volume and glomerular filtration. ${ }^{72,73}$ However, anti$\mathrm{Xa}$ levels are difficult to establish in daily monitoring. ${ }^{74}$

Administration of LMWHs is permitted if serum creatinine is less than $30 \mathrm{~mL} / \mathrm{min}$, as they are secreted by kidneys and patients weight is less than $150 \mathrm{~kg}$. If pregnant women weight is greater than $150 \mathrm{~kg}$, then anti-Xa levels monitoring should be performed more frequently to ensure the therapeutic effect or unfractionated heparin (UFH) must be administrated..$^{75}$

LMWHs are most active in a tissue phase and do not exert effects on coagulation factor IIa, for this reason monitoring of APTT is neither necessary nor useful during administration of LMWHs. Around the world, many LMWHs products with pharmacokinetic differences are available and their dose is highly product specific.

Administration of LMWHs has a longer duration of anticoagulant effect, more predictable therapeutic response (greater bioavailability), ease of administration (subcutaneous), safely administered in an outpatient setting, monitoring and less heparin-induced thrombocytopenia. Comparing the LMWHs with Unfractionated Heparin (UFH), the cost and longer half-life are disadvantages of LMWHs.

Unfractionated Heparin (UFH): In patients who may have immediate surgery, UFH may be preferred because it has shorter halflife and its result may be reversible with protamine compared with LMWHs. Unfractionated IV heparin is effective but for therapeutic effect is more difficult to titrate.

When UFH is administered in an acute VTE, the administration is given by IV bolus and is followed by IV infusion. The titration of dose is performed with aPTT moritoring. The laboratory determines the target of aPTT. To titrate the goal of aPTT, heparin infusion is typically increased or decreased by $10-30 \% .^{74}$

Hence patient's aPTT is therapeutic and stable; the UFH may be converted to either subcutaneous UFH or LMWH. The disadvantage of subcutaneous UFH is less predictable for anti-coagulation because the variability of the dose is not efficacious to maintain response comparing to $\mathrm{LMWH}^{74}$

\section{Despite of these, the cost of LMWHs prohibit their use in some countries}

When subcutaneous heparin is preferred, 500 units must be every 12 hours and six hours after the second dose aPTT must be checked. Increasing or decreasing the dose by $10-30 \%$ is necessary be achieved the therapeutic range. As long as aPTT is stable, the value is checked again in 3-4 days and at least weekly thereafter. ${ }^{74}$ 


\section{Coumarin and direct thrombin inhibitors}

Coumarin derivatives are contraindicated in pregnancy, classified as category $\mathrm{X}$ by FDA and cross the placenta.

Despite Warfarin is concerned safe for fetus at first six weeks of pregnancy, it produces embryopathy between six to nine weeks of pregnancy in $4-5 \%$ of fetuses. In this embryopathy have been described: midface hypoplasia, scoliosis, short proximal limbs, short phalanges, chondromalacia punctate and stippled chondral calcification. ${ }^{76,77}$ At any stage of pregnancy, warfarin may produce ophthalmologic and central nervous system abnormalities as well as fetus death. However, the most serious complication of warfarin administration is the fetal intercerebral hemorrhage which has related with its anticoagulant effect. ${ }^{78}$

All the anticoagulant drugs (Unfractionated heparin, LMWHs, Warfarin) are safe during breast feeding as they are secreted in breast milk in clinically significant amounts.

A pregnant patient with acute $\mathrm{PE}$ to achieve treatment doses with anti-coagulant drugs must initiate and continue for five days intravenous heparin, subcutaneous heparin or LMWH. $^{79}$ Subsequently, the therapy must be continued with either heparin or low-molecular weight heparin throughout the pregnancy and at least six weeks postpartum. But, unfractionated heparin and LMWHs may be required in higher doses during pregnancy depending on increased clearance and degradation.

During postpartum PE, warfarin may be administered. When intravenous heparin is initiated, the international normalized ratio (INR) determines its continuation and INR is necessary to be greater than 2.0 for two days or for five days of therapy, whichever is longer.

When an episode of VTE or PE during pregnancy is diagnosed, the patient has been considered as a hypercoagulable person and recommended to have anticoagulation therapy throughout the duration of pregnancy. During postpartum the anticoagulation therapy is continued at least six weeks, for three to six months whichever is longer.

In the days surrounding delivery, intravenous unfractionated heparin may be useful hence in a case of hemorrhage or demand of surgical intervention, its short half -life enables anticoagulation to be stopped quickly. When anticoagulation is strictly contraindicated, IVC filters can be placed and retrieved safely during the peripartum period. $^{80}$

\section{Labor and delivery}

Throughout the word, many pregnant women have deliveries under therapeutic anticoagulation, without delivery-related bleeding to be increased ${ }^{81}$ If regional anesthesia is going to be performed, anticoagulation must be discontinued temporarily for decreasing the risk of epidural or spinal hematoma.

When a prophylactic LMWH dose is administered, neuraoxial regional anesthesia should be withheld for 12 hours following the last one, or 24 hours after the last therapeutic dose. If prophylactic UFH is used in up to 5000 units twice daily, neuroaxial anesthesia is safe, but in higher doses is less known. ${ }^{81}$

At 36 weeks of pregnancy or sooner if there is the suspicion of early delivery, LMWH must be converted to subcutaneous UFH to permit regional anesthesia in labor. When UFH is used, at last weeks of pregnancy, a value of aPTT can be checked to ensure clearance before the neuraxial anesthesia.

Subcutaneous LMWH or UFH may be discontinued 24-36 hours prior to anticipate delivery, when it is planned.

When subcutaneous LMWH or UFH are discontinued, but prolonged periods without anticoagulation are undesirable, the patient can be anticoagulated with intravenous UFH because of it has shorter half-life. The intravenous UFH may be discontinued any time and an aPTT is checked to ensure clearance (Table 10).

Table 10 Suggested protamine dose for reversal of UFH and $\mathrm{LMWH}^{82}$

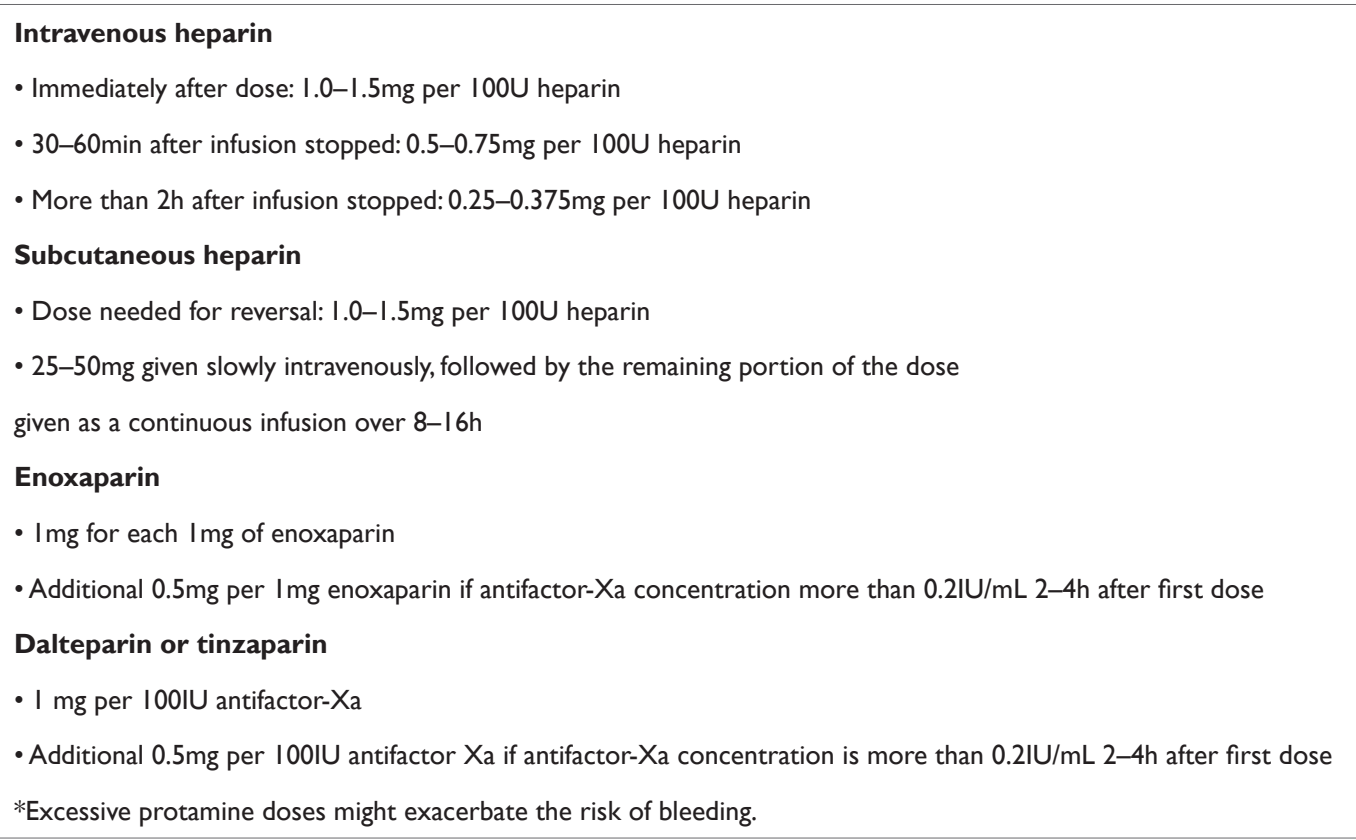


Table Continued....

\author{
Prophylactic LMWH, or UFH \\ (first trimester 5000IU; second trimester 7500IU; third trimester I0000IU) twice daily if LMWH is unavailable \\ No pharmacoprophylaxis Prophylactic LMWH, or UFH No pharmacoprophylaxis \\ (first trimester 5000IU; second trimester 7500IU; third trimester $10000 \mathrm{IU}$ ) twice daily if LMWH unavailable \\ VTE unprovoked and/or thrombophilia and/or hormonally provoked* \\ Previous VTE provoked by factors other than hormonal factors and no thrombophilia \\ Strong antenatal risk factors forVTE† Antepartum Other
}

VTE, venous thromboembolism; LMWH, low-molecular-weight heparin; UFH, unfractionated heparin

*Oral contraceptives, hormone replacement, or previous VTE during the antenatal period.

†Body-mass index more than $25 \mathrm{~kg} / \mathrm{m}^{2}$ and immobilisation, antithrombin deficiency, or combined thrombophilias.

\section{Postpartum}

When delivery is completed in woman with PE or VT, anticoagulation therapy must be initiated using subcutaneous UFH or LMWHs 4-6 hours after the vaginal delivery and 6-12 hours after cesarean delivery. ${ }^{83}$ After a neuraxial blockade, the ideal time for restarting prophylactic anticoagulation is unclear, but not sooner than 2 hours of epidural removal, ${ }^{84}$ and 12 hours after removal of the epidural catheter may be a reasonable approach. ${ }^{83}$

The duration and the kind of anticoagulation therapy depend on specific type of any thrombophilia and the time of thrombosis. In an acute episode of PE or VTE in pregnancy therapeutic anticoagulation is administered at least for 6 months, and for 6 weeks postpartum ${ }^{74}$ because in the postpartum period VTE is most likely to be apparent.

There is no evidence that heparins may produce any anticoagulation effects via the breast milk in the breast-fed infants.

\section{Prevention}

To prevent formation of thrombosis after delivery in low risk groups recommendations for early mobilization and stockings with graduated compression are effective, safe, and non-invasive methods. ${ }^{85}$

In pregnancy, antepartum and postpartum bleeding are around $0.4 \%$ and $1.5 \%$, respectively after pharmacological prophylaxis. ${ }^{86,87}$ Major bleeding and VTE have the same fatality rates. ${ }^{69,88}$

It has shown that more harm than good would be achieved with universal antepartum and postpartum prophylaxis, with around $0.05 \%$ absolute VTE risk for each time period. Patients with previous VTE present overall recurrence rates from $1.4 \%$ to $11.1 \%,{ }^{89,90}$ indicating that all women with a previous VTE, apart from those with VTE provoked by factors other than pregnancy or exogenous estrogen, should receive antepartum and postpartum prophylaxis. Thrombophilic conditions (inherited or acquired) suggest enhanced thrombosis risk. ${ }^{91,92}$

Thromboprophylaxis after caesarean section is not well established with adequately powered studies ${ }^{93}$ and meaningful conclusions cannot be reached. ${ }^{94}$ When there are risk factors for thrombosis such as body mass index $25 \mathrm{~kg} / \mathrm{m}^{2}$ or more and immobilizaton for longer than a week, thromboprophylaxis should be administrated as risk of VTE is very high. ${ }^{95}$

\section{Discussion}

Pregnancy is a period that a healthy woman is going to give life to her fetus. Sometimes it is unknown that she or her family disturbancies of coagulation factors. In many medical cases the doctor will be very lucky if the pregnant woman knows her or her family medical history. Other times there are coagulation disturbances in distant relatives that pregnant woman ignores their medical history. The thrombi produce severe medical signs of the body and are able to appear in any trimester of pregnancy without a specific cause. The physician is called to suspect and give prompt diagnosis and therapy to avoid the lethal condition. The lung thrombosis or pulmonary embolism is a rare disorder. This disorder has no specific clinical sings and requires specialized investigation to confirm the diagnosis. The loss of time for establishing a correct diagnosis is the cause that many pregnant patients die from misdiagnosed pulmonary embolism.

Delays in pulmonary embolism diagnosis are common despite the diagnostic advances and represent an important issue. Preventing idiopathic outpatient pulmonary embolism is difficult, if not impossible.

Administration of heparin or LMWHs can reduce the severity and incidence of death in these cases leading to a normal labor and a normal life of mother and neonate. The frequency of pulmonary embolism is avoided with the prevention of DVT in the lower extremities.

To avoid this severe condition, high risk population must be identified and safe- efficacious prophylactic measures with directions should be used.

Diagnostic tools and advances over the past several decades have significantly improved physicians' ability for differential and correct diagnosis of pulmonary embolism and administration for more suitable therapy without complications to the fetus or its mother.

Despite of these, several areas need further research and properly conducted therapeutic trials for sooner and correct diagnosis and treatment, avoiding the lethal complication for the pregnant woman and her fetus.

\section{Acknowledgments}

None.

\section{Conflicts of interest}

The authors declare no conflicts of interest.

\section{References}

1. Ozsu S, Oztuna F, Bulbul Y, et al. The role of risk factors in delayed diagnosis of pulmonary embolism. Am Emerg Med. 2011;29(1):26-32.

2. Marik PE, Plante LA. Venous thromboembolic disease and pregnancy. $N$ Engl J Med. 2008;359(19):2025-2033. 
3. Gherman RB, Goodwin TM, Leung B, et al. Incidence, clinicalcharacteristics and timing of objectively diagnosed venous thromboebolism during pregnancy. Obstet Gynecology. 1999;94:730 734 .

4. Toglia MR, Weg JG. Venous thromboebolism during pregnancy. $N$ Engl J Med. 1996;335:108-114.

5. Bates SM, Greer IA, Middeldorp S, et al. VTE, thrombophilia, antithrombootictherapy and pregnancy: antithrombolic therapy and prevention of thrombosis. $9^{\text {th }}$ ed, American College of Chest Physicians Evidence-Based Clinical Practice Guidelines. Chest. 2012;141(2 suppl) Qe691S-736S

6. Philipp CS, Faiz AS, Beckman MG, et al. Differences in thrombolic risk factors in black and white women with adverse pregnancy outcome Thromb Res. 2014;133(1):108-111.

7. Born D, Martinez EE, Almeida PA, et al. Pregnancy in patientswith prosthetic heart valves: the effects of anticoagulation on mather, fetus and neonate. Am Heart J. 1992;124(2):413-417.

8. Meschengieser SS, Fondevila CG, Santarelli MT, et al. Anticoagulation in pregnant women with mechanical heart valve prostheses. Heart. 1999;82(1):23-26

9. The Anticoagulation in Prosthetic Valves and Pregnancy Comsensus Report Panel and Scientific Rounttable. Anticoagulation and enoxaparin in patients with prosthetic heart valves and/or pregnancy. Fetal-Maternal Medicine Consesus Reports; 2002.

10. Ouellette DR, Harrington A, Kamangar N, et al. Pulmonary Embolism. 2018.

11. Heit JA. The epidemiology of venous thromboembolism in the community. Arterioscler Thromb Vasc Biol. 2008;28(3):370-372.

12. Scheider D, Lilinfeld DE, Im W. The epidemiology of pulmonary embolism:racial contrasts in I incidence and in-hospital case fatality. $J$ Natl Med Assoc. 2006;98(12)1967-1972.

13. Bernstein D, Coupey S, Schonberg SK. Pulmonary embolism in adolescents. Am J Dis Child. 1986;140(7):667-671.

14. Qaseem A, Snow V, Barry P, et al. Current diagnosis of venous thromboebolism in primary care: a clinical practice guideline from the American Academy of Family Physicians and the American College of Physicians. Ann Inter Med. 2007;146(6):454-458.

15. Qaseem A, Snow V, Barry P, et al. Current diagnosis of venous thromboebolism in primary care: a clinical practice guideline from the American Academy of Family Physicians and the American College of Physicians. Ann Fam Med. 2007;5(1):57-62.

16. Kucher N, Rossi E, De Rosa M, et al. Massive pulmonary embolism. Circulation. 2006;113(4):577-582.

17. Goldhaber SZ, Visani L, De Rosa M. Acute pulmonary embolism:clinica outcomes in the International Cooperative Pulmonary Embolism Registry (ICOPER). Lancet. 1999;353(9162):1386-1389.

18. Konstantinides SV, Torbicki A, Agnelli G, et al. 2014 ESC guidelines on the diagnosis and management of acute pulmonary embolism. Eur Heart J. 2014;35(43):3033-3069.

19. Meyer G, Planquette B, Sanchez O. Long-term outcome of pulmonary embolism. Curr Opin Hematol. 2008;15(5):499-503.

20. Worsley DF, Alavi A. Comprehensive analysis of the results of the PIOPED Study. Propspective investigation of pulmonary embolism diagnosis study. J Nucl Med. 1995;36(12):2380-2387.

21. Boyden EA. Segmental anatomy of the lungs: study of the patterns of the segmental bronchi and related pulmonary vessels. New York: McGrawHill; 1955:23-32.
22. Mitchell RN, Kumar V. Hemodynamic disorders, thrombosis, and shock. In: Kumar V, Cotran RS, Robbins SL, editors. Basic Pathology. 6TH ED. Philadelphia: WB Saunders; 1997:60-80.

23. Wharton LR, Pierson JW. Minor forms of pulmonary embolism after abdominal operations. JAMA. 1922;79(23):1904-1910.

24. Stein PD, Beemath A, Matta F, et al. Clinical characteristcs ofpatients with acute pulmonary embolism:data from PIOPED II. Am J Med. 2007;120(10):871-879.

25. Nelson-Piercy C, MacCallum P, Mackillop L. Green-top Guideline No. $37 \mathrm{a}$ - reducing the risk of venous thromboembolism during pregnancy and the puerperium. London: Royal College of Obstetricians and Gynaecologists; 2015.

26. Tapson VF. Acute pulmonary embolism. $N$ Endl $J$ Med. 2008;358(10):1037-1053.

27. Bauersachs RM, Manolopoulos K, Hoppe I, et al. More on the "ART" behind the clot: solving the mystery. J Thromb Haemost. 2007;5(2):438439.

28. Chan WS, Chunilal S, Lee A, et al. A red blood cell agglutination D-dimer test to exclude deep venous thrombosis in pregnancy. Ann Intern Med. 2007;147(3):165-170.

29. Boehlen F, Epiney M, Boulvain M, et al. D-dimer levels during pregnancy and the post-partum period. Results of two studies. Rev Med Suisse. 2005;1:296-298.

30. Turedi S, Gunduz A, Mentese A, et al. The value of ischemia-modified albumin compared with d-dimer in the diagnosis of pulmonary embolism. Respir Res. 2008;9:49.

31. Konstantinides S. Clinical practice. Acute pulmonary embolism. N Engl J Med. 2008;359(26):2804-2813.

32. Meyer T, Binder L, Hruska N, et al. Cardiac troponin I elevation in acute pulmonary embolism is associated with right ventricular dysfuction. $J$ Am Coll Cardiol. 2000;36(5):1632-1636.

33. Jimenez D, Uresandi F, Otero R, et al. Troponin-based risk stratification of patients with acute nonmassive pulmonary embolism: systematic review and metaanalysis. Chest. 2009;136(4):974-983.

34. Cavallazzi R, Nair A, Vasu T, et al. Natriuretic peptides in acute pulmonary embolism: a systematic review. Intensive Care Med. 2008;34(12):2147-2156.

35. Kucher N, Printzen G, Goldhaber SZ. Prognostic role of brain natriuretic peptid in acute pulmonary embolism. Circulation. 2003;107(20):25452547.

36. Kline JA, Zeitouni R, Marchick MR, et al. Comparison of 8 biomarkers for prediction of right ventricular hypokinesis 6 months after submassive pulmonary embolism. Am Hert J. 2008;156(2):308-314.

37. Dresden S, Mitchell P, Rahimi L, et al. Right Ventricular dilatation on bedside echocardiography performed by emergency physicians aids in the diagnosis of pulmonary embolism. Ann Emerg Med. 2014;63(1):1624.

38. Winer-Muram HT, Boone JM, Brown HL, et al. Pulmonary embolism in pregnant patients: fetal radiation dose with helical CT. Radiology. 2002;224(2):487-492.

39. Hurwitz LM, Yoshizumi T, Reiman RE, et al. Radiation dose to the fetus from body MDCT during early gestation. AJR Am J Roentgenol. 2006;186(3):871-876

40. Doshi SK, Negus IS, Odukok JM. Fetal radiation dose from CT pulmonary angiography in late pregnancy: a phantom study. Br J Radiol. 2008;81(968):653-658. 
41. Eskandar OS, Eckford SD, Watkinson T. Safety of diagnostic imaging in pregnancy. Part 1: X-ray, nuclear medicine investigations, computed tomography and contrast media. Obstet Gynaecol. 2010;12:71-78.

42. Remy-jardin M, Pistolesi M, Goodman LR, et al. Management of suspected acute pulmonary embolism in the era of CT angiography: a statement from the Fleidchner Society. Radiology. 2007;245(2):315-329.

43. Becattini C, Agnelli G, Vedovati MC, et al. Multidetector computed tomography for acute pumonary embolism: diagnosis and risk stratification in a single test. Eur Heart J. 2011;32(13):1657-1663.

44. Winer -Muram HT, Boone JM, Brown HL, et al. Pulmonary embolism in pregnant patients:fetal radiation dose with helical CT. Radiology. 2002;224:487-492.

45. Hiorns MP, Mayo JR. Spiral computed tomography for acute pulmonary embolism. Can Assos Radiol J. 2002;53:258-268.

46. Fedullo PF, Tapson VF. Clinical practice. The evaluation of suspected pulmonary embolism. $N$ Engl J Med. 2003;349:1247-1256.

47. Bettmann MA, Baginski SG, White RD, et al. ACR Appropriatenness Criteria acuute chest pain-suspected pulmonary embolism. J Thorac Imaging. 2012;27(2):W28-W31.

48. Gottschalk A, Stein PD, Sostman HD, et al. Very low probability interpretation of $\mathrm{V} / \mathrm{Q}$ lung scans in combination with low probability objective clinical assessment reliably excudes pulmonary embolism: data from PIOPED II. J Nucl Med. 2007;48(9):1411-1415.

49. Gupta A, Frazer CK, Ferguson JM, et al. Acute pulmonary embolism: diagnosis with MR angiography. Radiology. 1999;210(2):353-359.

50. Vanni S, Polidori G, Vergara R, et al. Prognostic value of ECG among patients with acute pulmonary embolism and normal blood pressure. Am J Med. 2009;122(3):257-264.

51. Ghada Bourjeily, Michael Paidas, Hanan Khalil, et al. Pulmonary embolism in pregnancy. Lancet. 2010;375(9713):500-512.

52. Russell JR, Stabin MG, Sparks RB, et al. Radiation absorbed dose to the embryo/fetus from radiopharmaceuticals. Health Phys. 1997;73(5):756769.

53. Chan WS, Ray JG, Murray S, et al. Suspected pulmonary embolism in pregnancy: clinical presentation, results of lung scanning, and subsequent maternal and pediatric outcomes. Arch Intern Med. 2002;162(10):1170 1175 .

54. Doyle N, Ramirez MM, Mastrobattista JM, et al. Diagnosis of pulmonary embolism: a cost-eff ectiveness analysis. Am J Obstet Gynecol. 2004;191(3):1019-1023.

55. Hopper KD, King SH, Lobell ME, et al. The breast: in-plane X-ray protection during diagnostic thoracic CT-shielding with bismuth radioprotective garments. Radiology. 1997;205(3):853-858.

56. Andreou AK, Curtin JJ, Wilde S, et al. Does pregnancy affect vascular enhancement in patients undergoing CT pulmonary angiography? Eur Radiol. 2008;18(12):2716-2722.

57. Pomp ER, Lenselink AM, Rosendaal FR, Doggen CJ. Pregnancy, the postpartum period and prothrombotic defects: risk of venous thrombosis in the MEGAstudy. J Thromb Haemost. 2008;6(4):632-637.

58. Ginsberg JS, Hirsh J, Turner DC, et al. Risk to the fetus of anticoagulant therapy during pregnancy. Thromb Haemost. 1989;61:197-203.

59. Ginsberg JS, Bates SM. Management of venous thromboebolism during pregnancy. J Thromb Haemost. 2003;1(7):1435-1442.

60. Van Dongen CJ, van den Belt AG, Prins $\mathrm{MH}$, et al. Fixed dose subcutaneous low molecular weight heparins versus adjusted dose unfractionated heparin for venous thromboembolism. Cochrane Database Syst Rev. 2004;(4):CD001100.
61. Pravinkumar E, Webster NR. HIT/HITT and alternative anticoagulation: current concepts. Br J Anaesth. 2003;91(4):676-685.

62. Warkentin TE, Levine MN, Hirsh J, et al. Heparin - induced thrombocytopenia in patients treated with low-molecular -weight heparin or unfractionated heparin. N Engl J Med. 1995;332:1330-1335.

63. Dolovich LR, Ginsberg JS, Dpiletos JD, et al. A meta-analysis comparing low-molecular-weight heparin with unfractionated heparin in the treatment of venous thromboembolism. Arch Intrn Med. 2000;160(2):181-188.

64. Devendra G, Morris T. Pulmonary embolism and deep venous thrombosis treated with either low molecular weight heparin or unfractionared heparin have the same incidence of hrombocytopenia. Chest. 2000;118:262.

65. Lindhoff -Last E, Nkov R, Misselwitz, Breddin HK, Bauersachs R. Incidence and clinical relevance of heparin -induced antibodies in patients with deep vein thrombosis treated with unfractionated or lowmolecular -weight heparin. Br J Haematol. 2002;118:1137-1142.

66. Schulman S, Beyth RJ, Kearon C et al. Hemorrhagic complications and thrombolytic treatment: American college of chest physicians evidence based clinical practice guidlines. Chest. 2008;133(6 Suppl):257S-298S.

67. Von Mandach U, Aebersold F, Huch A. Short-term low -dose heparin plus bedrest impairs bone metabolism in pregnant women. Eur J Obstet Gynecol Reprod Biol. 2003;106:25-30.

68. Guidlines on diagnosis and management of acute pulmonary embolism. Task Force on Pulmonary Embolism. European Society of Cardiology. Eur Heart J. 2000;21(16):1301-1336.

69. Hirsh J, Warkentin TE, Shaughnessy SG, et al. Heparin and lowmolecular-weight heparin: mechanisms of action, pharmacokinetics, dosing, monitoring, efficacy, and safety. Chest. 2001;119:64S-94S.

70. Bates SM, Ginsberg JS. How we manage venous thromboembolism during pregnancy. Blood. 2002;100(10):3470-3478.

71. Jacobsen AF, Qvigstad E, Sandset PM. Low molecular weight heparin (dalteparin)for thetreatment of venous thromboembolism in pregnancy. BJOG. 2003;110(2):139-144.

72. Casele HL, Laifer SA, Woelkers DA, et al. Changes in the pharmacokinetis of the low -molecular-weight heparin enoxaparin sodium during pregnancy. Am J Obstet Gynecol. 1999;18(5 Pt 1):1113-1117.

73. Ginsberg JS, Hirsh J. Anticoagulants during pregnancy. Annu Rev Med. 1989;40:79-86.

74. Bates SM, Greer IA, Pabinger I, et al. Venous thromboembolism, thrombophilia, antithrombotic therapy, and pregnancy: American college of chest physicians evidence-basedclinical practice guidelines. 8th ed, Chest. 2008;133(6 Suppl):844S-886S.

75. Paralkar VR, Rubin RN. Anticoagulation: An Update for Primary Care. Consultant. 2011;51(10).

76. Greer IA, Thomson AJ. Management of venous thromboebolism in pregnancy. Best Pract Res Clin Obstet Gynaecol. 2001;15:583-603.

77. Schaefer C, Hannemann D, Meister R, et al. Vitamin K antagonists and pregnancy outcome. A multi-centre prospective study. Thromb Haemost. 2006;95(6):949-957.

78. Greer IA. Thrombosis in pregnancy: maternal and fetal issues. Lancet. 1999;353(9160):1258-1265.

79. Buller HR, Agneli G, Hull RD, et al. Antithrombotic therapy for venous thromboembolic disease: the Seventh ACCP Conference on Antithrombotic and Thrombolytic Therapy. Chest. 2004;126:401S-428S.

80. Kocher M, Krcova V, Cerna M, et al. Retrievable Tulip Vena Cava Filter in the prevention of pulmonary embolism in patients with acute deep venous thrombosis in perinatal period. Eur J Radiol. 2009;70(1):165169. 
81. Dulitzki M, Pauzner R, Langevitz P, et al. Low molecular weigh heparin during pregnancy and delivery:a preliminary experience with 41 pregnancies. Obstet Gynecol. 1996;87(3):380-383.

82. Hirsh J, Bauer KA, Donati MB, et al. Parenteral anticoagulants: American college of chest physicians evidence-based clinical practice guidelines 8th ed. Chest. 2008;133(suppl 6):141S-1459.

83. James A, Committee on Practice Bulletins-Obstetrics. Practice Bulletin No 123: Thromboemolism in pregnancy. Obstet Gynecol. 2011;118(3):718-729.

84. Horlocker TT, Wedel DJ, Rowlingson JC, et al. Executive summary: regional anesthesia in the patient receiving antithrombotic or thrombolytic therapy: American Society of Regional Anesthesia and Pain Medicine Evidende-Based Guidelines. 3rd ed. Reg Anesth Pain Med. 2010;35(1):102-105.

85. Geerts WH, Bergqvist D, Pineo GF, et al. Prevention of venous thromboembolism: American college of chest physicians evidence-based clinical practice guidelines. 8th ed. Chest. 2008;133(6 Suppl):381-453.

86. Greer IA, Nelson-Piercy C. Low-molecular-weight heparins for thromboprophylaxis and treatment of venous thromboembolism in pregnancy: a systematic review of safety and efficacy. Blood. 2005;106(2):401-407.

87. Linkins L-A, Choi PT, Douketis JD. Clinical impact of bleeding in patients taking oral anticoagulant therapy for venous thromboembolism. A meta-analysis. Ann Intern Med. 2003;139(11):893-900.

88. Douketis JD, Kearon C, Bates SM, et al. Risk of fatal pulmonary embolism in patients with treated venous thromboembolism. JAMA $1998 ; 279(6): 458-462$
89. Pabinger I, Grafenhofer H, Kaider A, et al. Risk of pregnancy-associated recurrent VTE in women with a history of venous thrombosis. J Thromb Haemost. 2005;3(5):949-954.

90. Brill-Edwards P, Ginsberg JS, Gent M, et al. Safety of withholding heparin in pregnant women with a history of venous thromboembolism. Recurrence of Clot in This Pregnancy Study Group. $N$ Engl J Med. 2000;343(20):1439-1444.

91. Dizon-Townson D, Miller C, Sibai B, et al. The relationship of the factor $\mathrm{V}$ Leiden mutationand pregnancy outcomes for mother and foetus. Obstet Gynecol. 2005;106(3):517-524.

92. Middeldorp S, Meinardi JR, Koopman MMW, et al. A prospective study of asymptomatic carriers of the factor $\mathrm{V}$ leiden mutation to determine the incidence of venous thromboembolism. Ann Intern Med. 2001;135(5):322-327.

93. Gates S, Brocklehurst P, Davis LJ. Prophylaxis for venous thromboembolic disease in pregnancy and the early postnatal period. Cochrane Database Syst Rev. 2002;(2):CD001689.

94. Bates SM, Greer IA, Pabinger I, et al. Venous thromboembolism, thrombophilia,antithrombotic therapy, and pregnancy: American College of Chest Physicians evidence-based clinical practice guidelines. 8th ed Chest. 2008;133(suppl 6):844S-886.

95. Jacobsen AF, Skjeldestad FE, Sandset PM. Ante- and postnatal risk factors of venous thrombosis: a hospital-based case-control study. $J$ Thromb Haemost. 2008;6(6):905-912. 